\title{
Proposal for Multi-Messenger Observations of Radio Transients by Nasu and Ligo-Virgo
}

\author{
Kazuhiro Hayama ${ }^{1,3}$ and Kotaro Niinuma ${ }^{2}$ and Tomoaki Oyama ${ }^{3}$ \\ ${ }^{1}$ Max-Planck-Institut für Gravitationsphysik, D-30167 Hannover, Germany \\ email: kazuhiro.hayama@ligo.org \\ ${ }^{2}$ Graduate School of Science and Engineering, Yamaguchi University, \\ Yamaguchi 753-8511, Japan \\ ${ }^{3}$ National Astronomical Observatory of Japan, Mitaka, Tokyo 181-8588, Japan
}

\begin{abstract}
The gravitational waves radiated from explosions of compact objects are expected to be accompanied by other astronomical radiation such as radio flares, neutrinos and gamma-rays. In order to improve the detection of gravitational waves, mutual follow-up observations have been proposed between gravitational wave telescopes and other astronomical telescopes, known as "multi-messenger" observations. We propose multi-messenger observations using the LIGO, Virgo and Nasu Radio Telescopes.
\end{abstract}

Keywords. multi-messenger, gravitational waves, radio transients

\section{Introduction}

The Nasu radio telescope, located in Nasu, $160 \mathrm{~km}$ north of Tokyo, consists of eight spherical dish antennæ each of $20 \mathrm{~m}$ diameter, and a spherical dish antenna of $30 \mathrm{~m}$ diameter. Its observation frequency is $1.42 \mathrm{GHz}$. It is designed to detect transient radio signals and variable radio objects, and has detected more than 10 events since 2004 . Although the origin of these transient events is not known, one potential source is the radio remnants of compact binary mergers; they are prime sources of gravitational waves. A merger is expected to produce significant energetic outflows, whose optimal frequency is $\sim 1.4 \mathrm{GHz}$ (Naka \& Piran 2011) - the observing frequency of the Nasu telescope.

Searches for gravitational waves have been performed by $\mathrm{km}$-scale interferometetric gravitational-wave telescopes such as Ligo (Abbott et al. 2009a) and Virgo (Accadia et al. 2011). One of the main searches carried out is a blind search for transient gravitational waves, including binary neutron-star coalescence (Abbott et al. 2009b, 2009c). That type of search does not use astrophysical information from electro-magnetic observation. On the other hand, so-called "multi-messenger" observations, which use both gravitational-wave observations and other astronomical observations, have been performed to search for gravitational waves associated mainly with gamma-ray bursts (Abbott et al. 2010a, 2010b).

In this paper we propose multi-messenger observing of radio transients using the Nasu Radio Telescope and Ligo-Virgo.

\section{Overview of Waseda Nasu Pulsar Observatory}

\subsection{Specification of the observatory}

The Waseda Nasu Pulsar Observatory is located in Nasu, Tochigi Prefecture, Japan, $160 \mathrm{~km}$ north of Tokyo, at latitude $36^{\circ} 55^{\prime} 41^{\prime \prime} .3$ north and longitude $139^{\circ} 58^{\prime} 54^{\prime \prime} .3$ east. 
The transients monitoring array is chiefly composed of eight 20-m spherical dish antennæ (Daishido et al. 2000; Takeuchi et al. 2005). In order to search for radio transients or to monitor the radio variability of AGNs, those antennæ utilize four pairs of interferometers with an 84-m base-line. Four different declinations are monitored simultaneously with four fringe beams. Observations are performed as an earth-rotation drift-scan (Kuniyoshi et al. 2006). For any direction within $\pm 5^{\circ}$ of the zenith, a declination of $+32^{\circ}-42^{\circ}$ is observable by moving a symmetrical Gregorian sub-reflector. The half-power beamwidth is approximately $0^{\circ} .8$, and the fringe spacing approximately $10^{\prime}-11^{\prime} .5$, depending on the declination and the projected base-line (Kida 2008). The field of view is $0^{\circ} .8$ in latitude, $3^{\circ} .2$ in declination (Niinuma 2007b). The frequency of the observations is $1.42 \pm 0.01 \mathrm{GHz}$.

\subsection{Method for detecting radio transients}

If a point source passes through the fringe beam of the Nasu interferometers, the beam pattern (defined as the product of the primary beam and the fringe pattern, spaced at 40-46 seconds), appears in the data (Kuniyoshi et al. 2006; Takefuji 2007). The method of searching for radio transients is the same as for normal radio sources: the fringe signal is identified as coming from a transient source if the beam pattern appears clearly. The duration of all the radio transients detected at Nasu is at least 4 minutes. The fringe cycle provides further evidence that the signal originated from a celestial object.

\subsection{Potential of Nasu Observatory}

The spatial FFT observing system for direct imaging was developed in Daishido laboratory (Daishido et al. 2000; Nakajima 1993; Otobe 1994), and we have been carrying out test observations with this system at Nasu Observatory. When one can carry out a stable observation, it will be possible to

- obtain the dispersion measure within a bandwidth of $20 \mathrm{MHz}$; we can estimate the distance to the origin by assuming the column density of electrons, and

- detect radio sources with a time-resolution of 50 micro-seconds by observing the same object eight times in a day, about 25 seconds apart.

\section{Nasu and Ligo-Virgo: linked operations}

Multi-messenger observations with the Nasu radio telescope and LiGO-Virgo relax the detection threshold for gravitational-wave searches, and should improve the efficiency of detections. Fortunately, since several radio transients were detected by the Nasu instruments while Ligo and Virgo were also making observations in 2005-2007, we could carry out multi-messenger observations.

The multi-messenger observation has two modes. One is to follow up observations triggered by other astronomical detections. When a transient event is detected and recognized, information about the position and arrival time of the event is recorded. The follow-up observation of a gravitational wave associated with that event then incorporates that information in its search parameters. By noting any coincidences between the two observations, a low-threshold search can be performed that involves archived data, thus enabling the detection of a fainter gravitational wave signal than would result from the blind search. The other is a follow-up observation of an astronomical wave triggered by a gravitational wave. In typical explosions of compact objects such as merging binary neutron stars, electromagnetic waves are radiated for a duration of a few milliseconds to a few weeks, after any gravitational radiation (Hansen et al. 2001). When a gravitationalwave candidate is found the alert - including information about sky position and arrival 
time - is sent to other astronomical telescopes and follow-up observations are requested. The difference in the arrival times will vary according to frequency. In the case of a radio flare, the time difference is a few weeks, but as the durations of radio transients are a few days to a few weeks that is long enough to arrange follow-up observations. Since Nasu is observing continuously in a direction that is within \pm 5 degrees of the zenith, the discovery of radio transients is more likely from the region within the sensitivity of the Nasu telescope.

\section{References}

Abadie, J., et al. (2011), Physical Review D, 82, 102001

Abbott, B. P., et al. (2009a), Reports on Progress in Physics, 72, 7

Abbott, B. P., et al. (2009b), Physical Review D, 80, 062001

Abbott, B. P., et al. (2009c), Physical Review D, 80, 062002

Abbott, B. P., et al. (2010a), ApJ, 715, 1438

Abbott, B. P., et al. (2010b), ApJ, 715, 1453

Accadia, T., et al. (2011), Classical and Quantum Gravity, 28, 11

Daishido, T., et al. (2000), Proc. SPIE, 4015, 73

Hansen, B. M. S., et al. (2001), Mon. Not. R. Astron. Soc., 322, 695

Kida, S., et al. (2008), New Astronomy, 13, 519

Kuniyoshi, M., et al. (2006), PASP, 118, 901

Kuniyoshi, M., et al. (2007), PASP, 119, 122

Nakajima, J., et al. (1993), PASJ, 45, 477

Nakar, E. \& Piran, T. (2011), Nature, 478, 82

Niinuma, K., et al. (2007), PASP, 119, 112

Niinuma, K., et al. (2007), ApJ, 657, L37

Niinuma, K., et al. (2009), ApJ, 704, 652

Otobe, E., et al. (1994), PASJ, 46, 503

Takefuji, K., et al. (2007), PASP, 119, 1145

Takeuchi, H., et al. (2005), PASJ, 57, 815 